\title{
Neuropsicología cognitiva: Consideraciones metodológicas
}

\author{
LiLianne Manning \\ Neuropsychology Unit. The Radcliffe Infirmary, Oxford
}

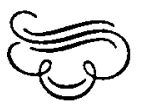

Resumen

\begin{abstract}
El presente trabajo es teórico y pretende ofrecer una revisión metodológica en una rama de la psicología que está en pleno auge, la neuropsicología cognitiva. El trabajo consta de los siguientes puntos: definición de la neuropsicología cognitiva y delimitación del concepto frente a la neuropsicología tradicional; relaciones entre la neuropsicología y la psicología cognitivas; métodos actuales en neuropsicología cognitiva, esto es, descripción y consideraciones de las distintas aproximaciones metodológicas; razones para el estudio de caso único, el método de la coberencia con el paciente, el método de la disociación en base a la bipótesis de la modularidad, el método de las constelaciones de síntomas y, por último, los requisitos necesarios para el sistemático estudio de caso único; críticas y conclusiones.
\end{abstract}

Palabras clave: Modelos congnitivos; lesión funcional; sistema normal/alterado; $\mathrm{N}=1$; modularidad; disociación; constelación de síntomas.

\section{Abstract}

A theoretical review on the methodology of cognitive sueropsychology is discussed. Cognitive neuropsychology is defined stressing its differences from the medical neuropsychology. Likewise, its common grounds with cognitive psychology are commented. Secondly, the main methodological approaches are considered, thus, the facts adduced to reject the group studies and the leading authors' views on the single patient methods: patient-theory coberence; symptom complexes; dissociations on the modularity account and the theoretically most rigorous single case claims. Finally, some criticisms and some conclusions are drawn.

Key words: Cognitive models; functional lesion; normal/impaired system; single-case; modularity; dissociation; sympton complexes.

Agradecimientos: El presente trabajo ha sido realizado gracias a la beca MEC/Fleming del Ministerio de Educación y Ciencia Español y del British Council. La autora expresa igualmente su agradecimiento al Dr. Allport (Dep. of Psychology) y al Dr. Marshall (Neuropsychology Unit), por sus valiosos comentarios. Las peticiones de ejemplares pueden dirigirse a Dr. L. Manning, Neuropsychology Unit. The Radcliffe Infirmary, Woddstock Road, Oxford OX2 6HE. United Kingdom. 


\section{INTRODUCCION}

Dentro de la neuropsicología clásica se procura responder a las preguntas planteadas sobre la sintomatología de un determinado paciente, con datos referentes a la localización de la lesión. Afirmar que un cuadro de jergafasia es el resultado de una lesión ocurrida en la porción posterior de la zona del lenguaje del hemisferio izquierdo, ejemplificaría el típico procedimiento tradicional, de corte médico de la neuropsicología. Por el contrario, desde una aproximación cognitiva, de corte eminentemente psicológico, la neuropsicología actual plantea lo siguiente:

- El patrón de alteración que presenta el paciente X, spuede ayudarnos de algún modo a comprender la organización normal de la mente y del cerebro?

Este segundo tipo de pregunta puede contestarse sugiriendo que el módulo que mediatiza el procesamiento psicológico que permite otorgar significado a la palabra hablada se ha alterado debido a una lesión. Este tipo de respuesta está totalmente basado en modelos cognitivos que se aplican a los casos de lesión cerebral con el resultado de un enriquecimiento bidireccional, que espero se haga patente a lo largo del presente trabajo.

Si bien ambos enfoques pueden ser válidos en sus propias perspectivas, únicamente el segundo, como es obvio, constituye el modo de investigar en neuropsicología cognitiva. Con ello pretendo ilustrar en pocas palabras la diferencia que existe entre neuropsicología tradicional y la cognitiva (un desarrollo amplio puede encontrarse en Manning, en prensa). La neuropsicología cognitiva (NPC) se basa en la explicación de síntomas que manifiestan los pacientes lesionados cerebrales, poniendo dichas explicaciones en términos de operaciones psicológicas alteradas, operaciones necesarias para el normal y eficaz proceso perceptivo, lingüístico, atencional, conceptual, mnésico, etc.

Es importante señalar que el dato anatómico, en neuropsicología cognitiva, es tomado en cuenta, pero nunca constituye el objetivo prioritario de la exploración; dicho en otras palabras, puesto que los déficits están relacionados con lesiones en determinadas áreas cerebrales, se mencionan los sitios de las lesiones, pero nunca se les da rango explicativo.

Coltheart (1986) señala un doble objetivo básico en NPC, en primer lugar los patrones de las ejecuciones cognitivas alteradas deben quedar explicados, así como el patrón de las ejecuciones cognitivas intactas realizadas por pacientes lesionados cerebrales. Tales explicaciones se realizan en términos de daño a uno o más componentes pertenecientes a una teoría o modelo del funcionamiento cognitivo normal. Consecuencia de este primer paso es la segunda parte del objetivo: llegar a conclusiones sobre el funcionamiento cognitivo normal.

En relación con todo ello, en NPC se sostiene que los patrones de síntomas observados no podrían ocurrir si el sistema intacto no estuviese organizado de una determinada manera. Los supuestos referentes a tal organización se apoyan -en el plano aplicado- en la constatación del fenómeno de disociación y -en el plano teórico- en la hipótesis de la modularidad (ambos serán brevemente desarrollados más adelante). 
En relación con todo ello, en NPC se sostiene que los patrones de síntomas observados no podrían ocurrir si el sistema intacto no estuviese organizado de una determinada manera. Los supuestos referentes a tal organización se apoyan -en el plano aplicado - en la constatación del fenómeno de disociación y -en el plano teórico- en la hipótesis de la modularidad (ambos serán brevemente desarrollados más adelante).

Hay que señalar, no obstante, que la NPC no es ni el único, ni el primer campo científico, en términos cronológicos, en el planteamiento de tales objetivos puesto que neurólogos, metodólogos y psicofisiólogos han comentado, desde hace un siglo, que el estudio de los procesos superiores en pacientes con daño cerebral podría abrir excelentes perspectivas en la comprensión de dichos procesos (véase Weiskrantz, 1968, o Kinsbourne, 1971, por ejemplo). ¿Qué aportaciones nuevas ofrece, entonces, la NPC? Sin lugar a dudas, el acercarse a los objetivos comentados, en base al estudio de caso único, enmarcado dentro del paradigma del procesamiento de la información.

\section{Psicología cognitiva y neuropsicología cognitiva}

Si la psicología cognitiva es el estudio de aquellos procesos mentales que hacen posible nuestro normal desenvolvimiento en el mundo que nos rodea y la neuropsicología es la ciencia que estudia la relación cerebro-conducta, la neuropsicología cognitiva representa la convergencia de estas dos disciplinas. La neuropsicología es cognitiva en la medida que trata de aclarar los mecanismos de las funciones cognitivas tales como pensar, leer, escribir, reconocer o recordar, utilizando la evidencia de la neuropatología (Campbell, 1987, en Ellis y Young, 1988).

El NPC recibió un importante impulso con trabajo de Shallice y Warrington en 1970, en el que se interpretaba el comportamiento mnésico alterado de un paciente lesionado cerebral basándose en las teorías cognitivas existentes por entonces, de la memoria a corto y largo plazo. El resultado de la exploración del paciente KF proporcionó una serie de datos empíricos que contradecían la teoría y el modelo de Atkinson y Shiffrin (1968), puesto que el paciente tenía preservada únicamente la memoria a largo plazo. Otra investigación de gran relevancia en la relación con las disciplinas que estamos viendo, constituyen el trabajo de Marshall y Newcombe (1973): Patterns of paralexia. Con dicha publicación se inició el estudio cognitivo del lenguaje en neuropsicología, al introducir, los autores, un modelo del procesamiento de lectura en sujetos normales. Se había iniciado un nuevo modo de trabajar en NPC. De hecho, à partir de estas investigaciones fueron introducidos los diagramas de flujo y se adoptó decididamente la perspectiva del procesamiento de la información.

Sin embargo, las relaciones entre la psicología cognitiva y la NPC no siempre han sido bidireccionales en términos de beneficios. Me explico: los psicólogos cognitivos no parecen tomar en cuenta el potencial que la NPC ofrece. Un ejemplo contundente lo constituye Neisser (1981), quien comenta, con claro pesimismo, que el trabajo de 100 años llevado a cabo en el campo de la memoria, no ha concluido más que evidencias que están al alcance de un niño de 10 años, o bien, sofisticadas teorías que no se tienen en pie en cuanto abandonan las cuatro paredes del laboratorio donde fueron concebidas mediante 
interminables variaciones experimentales. En ningún momento recurre Neisser a la investigación realizada con pacientes amnésicos. De haberlo hecho, habría vislumbrado alguna salida de tono más positivo, puesto que la significación de las diferencias individuales, el aprendizaje de la tarea, el esfuerzo requerido y la lista interminable de factores que afectan la ejecución en el laboratorio, se relativiza drásticamente frente a la importancia y especificidad de los déficits observados en casos de daño cerebral.

Otra teoría que podría beneficiarse de los hallazgos de la NPC es la de Paivio (Paivio, 1983; Paivio y te Linde, 1980) referente al doble sistema de codificación por el que los atributos perceptuales quedarían representados en un sistema pictórico mientras que los atributos conceptuales y lingüísticos quedarían representados en un sistema verbal. Por tanto, se sigue que el acceso más rápido a los atributos perceptuales será realizado a partir de cuadros o configuraciones (frente a palabras), mientras que un acceso más rápido a los atributos verbales se hará a partir de palabras. El color, es el trabajo de Paivio y te Linde (1980) queda definido como un atributo perceptual. Sin embargo, Beauvois (Beauvois, 1982; Beauvois y Derousné, 1980; Beauvois y Saillant, 1985) concluye que existe un efecto verbal superior al pictórico para el acceso al atributo color. Tal hallazgo fue realizado mediante el estudio exhaustivo de pacientes que sufrían afasia óptica para el color. (El argumento referente al atributo color, tal como lo acabo de exponer, acepta implícitamente un punto de partida de sistemas semánticos múltiples).

Por otra parte, la neuropsicología cuenta con evidencias sobre la organización de los hemisferios cerebrales que puede resultar de interés para el psicólogo cognitivo. En efecto, a partir de los análisis de los primeros niveles sensoriales y de la evidencia acumulada sobre los estadios superiores del procesamiento de la información, se ha puesto en evidencia una organización y un tipo de especialización independientes de los hemisferios cerebrales. Esta característica parece aplicable, sobre todo, a los lóbulos temporal y parietal. Los beneficios de un compromiso con la teoría de la especialización cerebral ya son evidentes en los análisis de los síndromes neuropsicólogicos complejos. Así, por ejemplo, la apraxia constructiva, fundamentalmente manifestada en la incapacidad del pacientes para dibujar, es frecuentemente descrita como el resultado de un daño cerebral, ya sea izquierdo o derecho. Ahora parece claro que si tal incapacidad proviene de un daño en el hemisferio derecho, la apraxia será el reflejo de una alteración en la percepción espacial, alteración que impide o dificulta la ejecución de un dibujo. Frente a ello, las lesiones del hemisferio izquierdo dan lugar, primariamente, a un déficit práxico: la incapacidad de llevar a cabo movimientos voluntarios.

Estos son algunos ejemplos de lo que puede aportar un visión complementaria de estas disiciplinas, con el consiguiente enriquecimiento de ambas.

\section{El método de la neuropsicología cognitiva}

Actualmente existe un total acuerdo en considerar que el método consistente en la categorización de síndromes (típicamente la aplicación de extensas baterías a todo tipo de pacientes con el objetivo de «etiquetar» el resultante perfil y agrupar de esa manera casos que, cualitativamente analizados, son absolutamente diferentes), tal como se ha venido haciendo en la neuropsicología 
de tradición médica, no solamente tiene una utilidad restringida a la comunicación interprofesional (remitir pacientes con sus respectivas etiquetas sindrómicas), sino que resulta perjudicial cuando lo que se quiere es investigar la estructura cognitiva de los procesos normales a partir de patrones de ejecución alterada (Caramazza y McCloskey, 1988). Voy a justificar mi acuerdo con tal afirmación: el punto de partida es, obviamente, la imposibilidad de obtener grupos variables para ser estudiados en neuropsicología. Primero, porque no existen síndromes homogéneos, es decir, cada paciente manifiesta un tipo de disfunción único; o dicho en otras palabras, en cada paciente se verán dañados distintos módulos y diferente subsistemas. Segundo, las diferencias observadas en la organización cerebral del sujeto sano no desaparecen en pacientes lesionados. Resulta, por consiguiente, imposible encontrar un conjunto de síntomas repetibles. Los dos puntos expuestos se pueden unir diciendo que la lesión nunca puede ser a priori, incide en un sistema con características propias y, por tanto, provoca un conjunto único de síntomas. Trabajar con la «media» de disfunción de un grupo no parece tener ningún aval científico, es más, constituye un sin sentido cuando la neuropsicología - de corte psicológico- trabaja con la perspectiva cognitiva, esto es, comprometida con el procesamiento normal, que, en definitiva, es lo que interesa conocer.

¿Cuál es entonces el camino que hay que seguir, una vez descartadas las categorías anatomo-clínicas? En general, los autores más influyentes en NPC (Morton y Patterson, 1980; Caramazza, 1984, 1986; Caramazza y McCloskey, 1988; Marshall, 1986; Howard, 1987; Ellis y Young, 1988, entre otros) abogan por el estudio de caso único como método adecuado. El estudio $\mathrm{N}=1$ implica contar con una línea base de datos a través de tests estandarizados y a partir de esa línea base todas las pruebas son diseñadas y construidas para cada paciente con vistas a testar una determinada hipótesis respecto a una alteración dada. Los resultados deben ser estadísticamente analizados y aquellas tareas que comporten una especial relevancia teórica, deberán ser administradas en más de una ocasión con el fin de establecer la replicabilidad de sus resultados. Asimismo, las conclusiones teóricas deben basarse en los datos obtenidos en más de una tarea.

El estudio de caso único ha sido realizado desde distintas aproximaciones epistemológicas y, por consiguiente, con distintas guías de aplicación cognitiva - del lenguaje en este caso- fue el publicado en 1980 por Coltheart, Patterson y Marshall Deep Dislexia. Este texto cobra un interés muy especial en las consideraciones metodológicas de la NPC porque ofrece los tres métodos que se puede seguir cuando se trabaja con caso único. Voy a comentarlos brevemente.

Morton y Patterson (1980), en primer lugar, representan la aproximación de rechazo radical a las entidades nosológicas. Ofrecen un modelo al que se van a referir para interpretar los datos que el paciente aporta, sin añadir ningún otro elemento teórico. Estos autores omiten toda opinión referente a la localización neuronal de los módulos que componen el diagrama, señalando que su interés radica en la identificación de componentes de comportamiento, no su localización. Sus informes de caso único tiene la exigencia prioritaria de ser coherentes solamente con el caso descrito. En sus palabras: "Aquello que va a decidir la descripción apropiada, dentro de un modelo determinado, va a ser el modelo de los síntomas" (p. 97) (Volveré a este enfoque más adelante).

El segundo acercamiento es planteado por Warrington y Shallice (1980) 
en base a la disociación, doble disociación, conjunto de disociaciones y - si bien reconociendo su menor grado de fiabilidad - la asociación de síntomas.

Desde un planteamiento de clínica diaria el estudio $\mathrm{N}=1$ se basa en un concepto ya antiguo en neuropsicología, la disociación (Teuber, 1955). Por disociación se entiende que si el paciente $\mathrm{X}$ tiene alterado el procesamiento de la tarea 1 , pero realiza correctamente la tarea 2 , se puede decir que muestra una disociación entre tales tareas dando lugar al postulado de que el sistema cognitivo normal debe estar organizado de manera que la ejeución de la tarea 1 se lleva a cabo independientemente del procesamiento de la tarea 2 . La doble disociación es la versión más exigente del mismo concepto, tendente a evitar los errores que la simple disociación pueda provocar al inferir sobre un cerebro intacto. Teuber lo explica así: «Para demostrar la especificidad del déficit discriminativo visual, necesitamos hacer algo más para demostrar que la discriminación en otra modalidad, por ejemplo, la somestésica, está preservada. Tal disociación simple puede indicar meramente que la discriminación visual es más vulnerable a las lesiones del temporal que la discriminación tactil. Este sería un caso de jerarquía funcional, más bien que un caso de localización separada. Lo que se requiere para obtener una prueba concluyente es la "doble disociación", esto es, obtener evidencia respecto a que la discriminación tactil puede ser alterada por cualquier otra lesión sin que se produzca déficit en las tareas visuales $y$ en un grado comparable en severidad al supuesto déficit visual consiguiente a lesiones temporales» $(1955$, p. 283). Hay que añadir un par de puntualizaciones, en primer lugar, la "preservación» no tiene por qué ser absoluta, basta una ejecución significativamente mejor en una tarea frente a otra, para poder concluir una disociación. En segundo lugar, hay que tener en cuenta que algunas veces pueden aparecer dos funciones alteradas, no porque la una dependa de la otra, sino porque ambas dependen de una tercera, o bien porque - sin existir ninguna relación funcional entre ellas - pueden verse simultáneamente alteradas porque sus respectivos tractos nerviosos, aunque separados en términos neuronales, pueden tener un recorrido tan cercano que resulten dañados por la misma lesión.

Una parte importante de base teórica que subyace al método de la disociación en NPC ha sido puesta a punto tras relacionar los datos empíricos de la disociación con la hipótesis de la modularidad. A fin de que los comentarios sobre dicha relación resulten claros, voy a resumir lo más importante - para la NPC - de la modularidad, recordando previamente que la NPC, como toda ciencia, se apoya en parte en un conjunto de postulados que no se pueden probar (Lakatos, 1974), siendo la modularidad uno de tales postulados, ya que por su naturaleza no puede ser testada directamente.

La modularidad es un principio opuesto a la equipotencialidad y como tal es sostenido por varias disciplinas como, por ejemplo, la informática, la linguística, la fisiología, la psicología. El argumento más básico lo ofrece la informática: «Cualquier computación importante en tamaño debe ser dividida e implementada como una colección de subpartes que sean más pequeñas y tan independientes las unas de las otras como el prograna en general lo requiera. Si el proceso no es diseñado de esa manera, cualquier cambio en un lugar determinado va a tener consecuencias en muchos otros sitios» (Marr, 1976, p. 485). En relación con lo dicho por Marr, pero en el dominio de la lingüística, Chomsky (1980) comenta que la manera más simple de explicar la independencia de nuestra competencia lingǘstica en un dominio particular, es asumir que se 
trata del producto de un subsistema que es $-\mathbf{a}$ su vez - relativamente independiente de otros subsistemas. Por su parte, Cowey (1982), dentro de la fisiología, ofrece uno de los argumentos más aceptados de modularidad: cada neurona, según este autor, necesita, para poder codificar un rasgo, tener conexiones con otras neuronas que analicen el mismo atributo. Llevar a cabo todas las operaciones referente a diferentes rasgos, en las mismas áreas cerebrales, provocaría una enorme complicación de los mecanismos encargados de localizar las conexiones adecuadas para cada demanda durante el desarrollo. Por el contrario, la especialización por zonas, implica que las columnas de neuronas pueden conectar con todas aquellas neuronas vecinas que realizan análisis de aspectos similares o relacionados.

Así pues, la noción de que existen subsistemas anatómicamente diferenciados que pueden realizar microfunciones separadas puede constituir la base explicativa del hecho de que diferentes regiones corticales respondan diferencialmente y de que tales zonas posean determinadas propiedades neuroanatómicas y neurofisiológicas. Quizá uno de los campos de la neurociencias en el que el concepto de modularidad ha demostrado una enorme utilidad es el de la visión en el que se ha trabajado, por ejemplo, sobre las propiedades de células nerviosas selectivamente sensibles al color (Zeki, 1980).

Uno de los ejemplos prototípicos que se ofrece en psicología para apoyar el principio de modularidad es el ampliamente conocido trabajo de McLeod, McLaughlin y Nimmo-Smith (1985).

La hipótesis de la modularidad aplicada a la neuropsicología puede resultar fructífera en términos de mediación entre el paciente lesionado cerebral y la comprensión de los procesos normales. En este sentido cabe dos posibilidades. Muy brevemente resumidas las dos posturas son las siguientes: Marr (1982, entre otros) sugiere que la comprensión de un sistema normal debe basarse en la delimitación del modo de trabajar de los hipotéticos subsistemas; exigencia que puede ser análoga a la pretensión de conocer cómo trabaja el bardware de un ordenador mediante la observación del disfuncionamiento del programa (del que nada se conoce) causado por un fallo mecánico del aparato; la tarea parece ardua, sino imposible. La otra alternativa enfatiza la delimitación de la organización modular mediante el aislamiento de los subsistemas que se presten a ello. En esta segunda alternativa se basa la «arquitectura funcional» del sistema de procesamiento del lenguaje, que es la que se sigue en NPC.

Si relacionamos la hipótesis de modularidad con la disociación, llegamos al postulado siguiente: las habilidades cognitivas están mediatizadas por un amplio número de procesos o sistemas cognitivos capaces de dañarse independiente. En este sentido se puede concebir un conjunto de módulos para llevar a cabo una serie de tareas con el supuesto básico de que el carácter discreto de los módulo hace que la lesión cerebral afecte a algunos de ellos, dejando los demás preservados o relativamente preservados.

Se ha relacionado la modularidad con la disociación por la importancia que tal relación cobra dentro de la metodología cognitiva. En efecto, si podemos hablar en términos de subsistemas, podremos afirmar que un caso clínico en el que se constata una doble disociación permite aislar determinados módulos o subsistemas, puesto que «si el proceso $X$ está intacto donde el proceso $Y$ está severamente comprometido o ausente $y$, especialmente, si se encuentra el caso inverso, en otro paciente, bay razón para creer que $X$ e $Y$ reflejan diferentes mecanismos subyacentes en estado normal» (Marin y col., 
1976, p. 869). En términos generales, los investigadores otorgan a la doble disociación una importancia crucial en la relación entre los datos empíricos y los subsistemas aislables.

Hay que señalar, en relación con las asociaciones, que existe una asimetría entre éstas y las disociaciones. Si un paciente manifiesta una asociación entre los tipos de déficits, mientras que otro paciente muestra una disociación entre los mismos déficits, con la consiguiente preservación de una de las capacidades, entonces la disociación - sugieren Warrington y Shallice (1980) - es el resultado de una lesión que ha afectado sólo un lado de la «linea funcional» del sistema modular. Frente a ello, una asociación resultaría de un tipo de lesión con las mismas características anatómicas necesarias para abarcar ambos lados de la línea funcional.

Hasta ahora he relacionado la disociación -fenómeno empírico- con la modularidad -aspecto inferencial, teórico-. El lector se puede preguntar qué tipo de consideraciones merece la disociación concebida dentro de una organización no modular, esto es equipotencial. En este sentido hay, en mi conocimiento, un solo trabajo (Wood, 1978), que es una revisión crítica de la metodología en NPC, en la cual se adelanta la hipótesis de que un sistema prácticamente equipotencial podría manifestar doble disociación al ser dañado. No voy a comentar, sin embargo, dicho trabajo, puesto que ha sido a su vez severamente criticado por Shallice (1988), quien detectó errores en los postulados de Wood, por lo que, evidentemente, no se lo puede tomar como una referencia válida. Parece, pues, que sólo queda discurrir lógicamente: El análisis de la acción de masa de Lashley (1929) y la coherencia con lo que tal principio implica, conducen absolutamente a la conclusión de que un sistema equipotencial no podrá dar lugar a una doble disociación, puesto que los efectos de todas las lesiones en todas las tareas pueden ser ordenados en una dimensión única que combine el tamaño de la lesión con la sensibilidad de la tarea. La ejecución en todas las tareas empobrecería gradualmente y de forma equivalente, a medida que la lesión aumentara en extensión. En tal cuadro parece imposible concebir una disociación. Frente a ello, el dato empírico, de clínica diaria, es que los pacientes presentan claras e irrefutables disociaciones y dobles disociaciones.

La tercera aproximación metodológica de la NPC presente en Deep Dislexia es sostenida por Coltheart (1980) y Marshall y Newcombe (1980) y puede ser denominada "método de las constelaciones de síntomas». Los autores nombrados, en claro contraste con Morton y Patterson (1980) intentan realizar generalizaciones empíricas inter-pacientes y utilizar métodos de selección de pacientes que no puedan ser explicados por las teorías existentes, en otras palabras; sugieren que la NPC avanzará con el estudio exhaustivo de casos capaces de falsear teorías.

El modo de realizar la aproximación de constelación de síntomas en NPC puede quedar más claro comentando el área en la que más se ha utilizado (al menos en mi conocimiento) el método que estamos viendo. Ante la constatación de errores semánticos de lectura (esto es, la dislexia profunda, en la que el paciente ante la palabra escrita "canario» lee 'periquito'), los autores tratan de averiguar qué otros síntomas acompañan invariablemente tal manifestación patológica a fin de identidicar el conjunto de síntomas asociados. En esta línea, Marshall y Newcombe (1980) postulan cuatro factores cuya co-ocurrencia se produce cuando el paciente presenta errores semánticos: 
1. Errores derivacionales (ante la palabra escrita «nacimiento», el paciente lee 'nacido').

2. Errores sintácticos («ratón» - 'razón').

3. Errores sintácticos («por» $\rightarrow$ ' $y$ ').

4. Imposibilidad de leer no-palabras, con el resultado de que cuando se presenta una no-palabra, el paciente va a convertirla en la palabra real morfológicamente más cercana («lumedo» $\rightarrow$ 'búmedo').

Dada la aparición concomitante de estos cuatro tipos de errores con los errores semánticos, Marshall y Newcombe plantean la posibilidad de que la lesión, en estos casos, esté descubriendo un sistema de lectura neuronalmente intacto - si bien subsidiario - en el hemisferio derecho que no se pondría de manifiesto en circunstancias normales, pero que la lesión ocurrida hace que tal sistema se ponga en funcionamiento.

Los autores dejan su postura bien definida comentando que la dislexia profunda no es un síndrome, sino la alteración de un mecanismo subyacente que se manifiesta en un conjunto de alteraciones visibles y coherentes. El método de las constelaciones de síntomas resulta especialmente interesante teniendo en cuenta que: «La lógica de cualquier análisis más exhaustivo de dislexia profunda, en términos de constelación de sintomas, parece presuponer cierta regularidad estadistica básica en los tipos de errores que aparecen o que no aparecen juntos" (Marshall y Newcombe, 1980, p. 3).

Además de estas tres ya clásicas aproximaciones, vamos a ver con algún detalle la aportación más reciente de Caramazza (1984, 1986; Caramazza y McCloskey, 1988; Oslen y Caramazza, en prensa) recordando que el citado autor es, posiblemente, uno de los neuropsicólogos más implicados en cuestiones metodológicas en NPC. Su aportación se dirige, como las tres anteriores, a lograr el objetivo de comprender mejor el funcionamiento normal del cerebro a partir de patrones de ejecución alterada por lesión, pero lo hace de manera más sistemática proponiendo un método muy riguroso en el estudio de cáso único, a partir de cuatro requisitos básicos (inicialmente expuestos en 1986):

1. ${ }^{\circ}$ Una hipótesis referida a la ejecución de una determinada tarea por un sujeto normal.

2. La hipótesis sobre el modelo normal alterado en caso de lesión cerebral.

3. ${ }^{\circ}$ El principio de sustractividad por el que el comportamiento del paciente refleja directamente la hipótesis del punto primero, sometida a la hipótesis del punto segundo.

4. ${ }^{\circ}$ El principio de universalidad por el que todos los sistemas cognitivos son básicamente idénticos.

Estos requisitos constituyen la base del trabajo metodológico del autor, quien establece que el interés de estudiar el comportamiento superior alterado en pacientes con lesión cerebral radica en el hecho de que tales pacientes ofrecen una única oportunidad de explorar el sustrato neuronal del procesamiento cognitivo humano. Por otra parte señalan que, desde un punto de vista pragmático, la utilización de déficits cognitivos, tanto para informar como para delimitar las teorías sobre el procesamiento cognitivo normal, proviene de la observación de que el daño cerebral no produce una pérdida 
indiferenciada de habilidades cognitivas, sino una pérdida selectiva de algunas habilidades. "Afortunadamente para nuestra empresa, el daño cerebral puede tener como resultado unos modelos de disfunción altamente selectivos, que reflejan, presumiblemente, la estructura componencial de los sistemas cognitivos» (Caramazza y McCloskey, 1988, p. 518). En base a ello, los autores postulan que las inferencias válidas que se realicen sobre la estructura cognitiva de sistemas normales, a partir de modelos de comportamiento alterado, serán posibles únicamente en estudios de caso único, con la consiguiente necesidad de perfeccionar el método, teniendo en cuenta que la teoría que sustenta dicho método queda plasmada en los diagramas de flujo que constituyen los modelos de procesamiento de las funciones superiores. Así pues, se debe articular la arquitectura funcional del sistema cognitivo (la arquitectura funcional emerge de la hipótesis de la modularidad previamente comentada), con el detalle necesario que permita identificar módulos de procesamiento con su aparataje computacional y su estructura interna. Asimismo, es necesario identificar la organización de esos módulos cuando se produce un proceso muy específico (por ejemplo, producción de frases).

Veamos, a continuación qué tipo de premisas científicas permiten llevar a cabo esta aproximación. El razonamiento que se inicia en el comportamiento alterado y llega al procesamiento normal, incluye los siguientes puntos:

1. La ejecución cognitiva normal $(\mathrm{O})$ es el resultado de un conjunto de componentes de procesamiento que forman el sistema cognitivo $M$.

2. Las posibles formas de alteración cognitiva están limitadas por la naturaleza de esos sistemas cognitivos normales. Esto significa que el comportamiento cognitivo alterado $\left(\mathrm{O}^{*}\right)$ refleja la actividad de un sistema cognitivo funcionalmente lesionado $\mathrm{M}+\mathrm{L} \rightarrow \mathrm{O} *$.

3. La actitud investigadora básica en NPC implica determinar, para cada paciente, la existencia o no existencia de modificaciones del sistema cognitivo, lo cual equivale a delimitar una disfunción que pueda explicar el modelo $\left(\mathrm{O}^{*}\right)$ de ejecución cognitiva alterada.

El punto crítico en este recorrido es que $\mathrm{L}$-disfunción o lesión funcional - que relaciona la ejecución real de un paciente determinado con un modelo congnitivo, nunca viene determinada a priori, sino que debe ser inferida a partir, precisamente, de la ejecución del paciente; este sería el razonamiento definitorio de la metodología a seguir en NPC.

Hasta aquí, la aportación de Caramazza tiene mucho en común con el quehacer de los neuropsicólogos cognitivos. Sin embargo, el autor que estamos siguiendo, obtiene, a partir de los puntos comentados, una serie de conclusiones sobre la manera de proceder, a fin de llegar a una mejor comprensión del funcionamiento normal, que no son compartidas por otros autores. Así, según Caramazza (1986), cada paciente debe ser tratado como una prueba de la teoría, por lo que es sumamente importante contar con un modelo cognitivo suficientemente detallado como para ir situando en él las ejecuciones alteradas y las ejecuciones preservadas de cada paciente (ésta y las siguientes conclusiones, las comento más adelante). Por otra parte, Caramazza considera que la doble disociación no tiene más relevancia teórica que cualquier otro hallazgo a que conduzca el estudio de $\mathrm{N}=1$ y que la 
replicabilidad directa de un caso es imposible en neuropsicología, añadiendo que tal imposibilidad carece de importancia, ya que el objetivo mismo de la NPC es alcanzable sin dicha condición. Por último, respecto al ya comentado estudio de grupos, señala y reitera la imposibilidad de obtener una base de datos válida a partir de los mismos, para inferir sobre el funcionamiento del sistema intacto.

Las consideraciones en torno a lo que hasta aquí se ha comentado llevan -en primer término- a sugerir que si partimos de la base de $\mathrm{N}=1$ en tanto que método de estudio que exige que un conjunto de teorías sean comparadas con una amplia serie de casos estudiados, de manera que puedan realizarse comparaciones inter-paciente, parece evidente que la aproximación de Morton y Patterson (1980) no ofrece claras posibilidades de progreso. Frente a ello, las otras dos perspectivas presentes en Deep Dislexia pueden conducir a comparaciones inter-paciente, de manera que cada uno no constituya en sí mismo una teoría y un modelo. Por otro lado, estas dos aproximaciones permiten que el objetivo científico en general, esto es, corroboración, descubrimiento o falsación, pueda ser alcanzado (como es lógico, en neuropsicología se asume que sólo la falsación y el descubrimiento podrán ser alcanzados) al seleccionar aquellos pacientes cuyo comportamiento tras la lesión cerebral sea relevante para falsar una teoría. En este sentido, el estudio $\mathrm{N}=1$ no parece plantear insuficiencia metodológica. El problema, al menos por el momento, es de orden práctico, las teorías son escasas y no todas las que existen han alcanzado un nivel adecuado de desarrollo.

En relación con la aproximación de Caramazza, que podríamos llamarla "caso único a ultranza", lo fundamental es resaltar la dificultad de contrastar sus postulados. A nivel más detallado, por otra parte, sus afirmaciones son criticables: su consideración respecto de la doble disociación implica, en mi opinión, cerrar las posibilidades más sólidas - en tanto que validez- del método $\mathrm{N}=1$.

Un punto crucial en la teoría de Caramazza (y también en la de Morton y Patterson, 1980) se refiere a la importancia que se debe dar a la coherencia paciente-teoría-modelo. Este punto ha sido fuertemente criticado por Shallice (1988), quien puso de relieve el peligro de hacer desaparecer la NPC en un mar de datos; extremo que puede ocurrir si, siguiendo los postulados de Caramazza, convertimos a cada paciente en una teoría. En este punto, Caramazza es, no obstante, defendido por algunos neuropsicólogos congnitivos, así, por ejemplo, Marshall (en comunicación personal, Oxford, 1989) sugiere que - precisamente siguiendo esos postulados - cada paciente vendría a confirmar parte de la teoría.

Bub y Bub (1988) documentan la crítica a Caramazza proporcionando un ejemplo muy interesante: En primer lugar, aclaran que si bien un estudio estadístico de pacientes lesionados cerebrales no puede pretender detectar una lesión funcional (L), ello no quiere decir que a partir de esta observación deba concluirse que los estudios de grupo no pueden probar ninguna hipótesis relacionada con determinadas propiedades globales del sistema normal de procesamiento de la información. Así, la denominación de objetos que depende de un conjunto de conocimientos jerárquicamente organizados (desde unos rasgos semánticos generales, hasta unos rasgos específicos), sugiere que los pacientes anómicos - como grupo- (véase Manning, 1988) deben ser sensibles a la distinción entre las descripciones subordinadas y 
superordinadas de objetos, de modo que los resultados estadísticos serán relevantes respecto de la hipótesis de funcionamiento general del sistema. De hecho, también un resultado nulo puede ser relevante en relación con hipótesis del funcionamiento normal del sistema. Así, podemos pensar -comentan Bub y Bub (1988)-- que los pacientes disléxicos (véase Coltheart, 1980), como grupo, no discriminan perfectamente entre palabras y no-palabras. Este resultado podría ser relevante para la hipótesis que establece que la lectura depende de unos procedimientos diferentes para items léxicos y no-léxicos, que funcionan en paralelo. Este ejemplo, se habrá dado cuenta el lector, es mucho más controvertible que el anterior.

Uno de los puntos de apoyo reiteradamente esgrimido por Caramazza siempre que en sus distintos escritos postula que la única manera de estudiar NPC es mediante el caso único, es que la lesión funcional L, es el factor crucial para analizar la ejecución del paciente $y$, a partir de tal ejecución, comprender $\mathrm{M}$; esto es, el modelo de funcionamiento normal. La crítica más directa a tal postulado ha sido formulada por Whitaker y Slotnick (1988), quienes señalan que $\mathrm{L}$ es uno de los factores, no el único factor. Otros factores a considerar serían las diferencias individuales premórbidas (¿posible?). Asimismo, habrá que considerar las variaciones de la interacción entre el modelo normal individual y la lesión funcional ocurrida. Esto último significa que el sistema dañado es, en parte, el resultado de la reacción del organismo a la lesión. Por consiguiente, para que el ideal metodológico de Caramazza sea posible, hay que estudiar, además de L, todos los otros factores. Implícita en este argumento está la crítica al principio de universalidad (el cuarto requisito básico de Caramazza, 1986). McCloskey y Caramazza (1988) responden a tal crítica mostrándose de acuerdo: «... el supuesto de universalidad no es aplicable a todos los supuestos del sistema congnitivo... Debemos estar siempre preparados para revisar basta los más básicos supuestos sobre los que basamos nuestro trabajo" (p. 597). Sin embargo, los autores, tras un extenso análisis de cuáles serían las consecuencias de rechazar dicho principio concluyen que: «Así, en nuestra opinión, el supuesto de universalidad debe ser considerado un supuesto defectuosò en la investigación cognitiva, al menos respecto a los mecanismos subyacentes a las funciones cognitivas básicas tales como percepción, memoria y precesamiento del lenguaje» (p. 598)

Finalmente Whitaker y Slotnick (1988) critican a Caramazza el hecho de olvidar, en su teorización, factores tan importantes como son las estrategias y/o los mecanismos compensatorios con los que el paciente se enfrenta a los efectos de su lesión cerebral. McCloskey y Caramazza (1988) rechazan de plano tal crítica recordando que sus modelos incluyen diversas rutas de posible ejecución.

\section{Conclusiones}

Una vez expuestos los caminos que actualmente se recorren en NPC, y tras haber comentado las críticas que una de las aproximaciones, sobre todo, ha recibido, cabe preguntarse si la NPC, tal como ha sido planteada, puede pretender avanzar en el conocimiento del sistema normal. Seidenberg 
(1988) ha contestado negativamente a esta pregunta proponiendo una analogía (o por lo menos, lo que Seidenberg cree que es una analogía): las dos autopistas de la ciudad $\mathrm{X}$ que conducen al aeropuerto están bloqueadas por una tormenta de nieve. Cómo llegue, a pesar de ello, la gente al aeropuerto o cómo fracase en su intento de llegar, nos ofrecerá un panorama de vías y calles laterales utilizadas en caso de bloquearse las principales, pero no nos dirá nada sobre las autopistas en sí. En resumen, la ejecución de pacientes lesionados cerebrales no nos conduce al conocimiento del sistema normal. Me parece que el ejemplo elegido por Seidenberg no es comparable con el análisis de vías del procesamiento funcional, únicamente tienen en común el vocablo "vía" en tanto que recorrido. Es evidente que el estudio de los recorridos que se realicen por las calles laterales no nos dirá nada sobre las vías principales, en el ejemplo que pone el autor, lo que resulta mucho menos evidente es que en neuropsicología contemos ya con vías laterales y principales tan claramente delimitadas, y si no contamos con ellas, la analogía no es válida.

Seidenberg (1988) también afirma que la NPC no puede ir más allá de lo que una simple intuición puede ofrecer. Mi opinión al respecto es bien diferente. Estoy convencida que una intuición, aún siendo brillante, habría fracasado en la delimitación de numerosos conocimientos que en base a los modelos y al proceder de la NPC se han establecido. Voy a poner ejemplos: el análisis de las palabras se realiza mediante subsistemas funcionalmente diferenciados. Así, leer una palabra escucharla y comprender su significado constituyen tres fenómenos llevados a cabo - tal y como lo demuestran los pacientes - por distintos mecanismos. Otro ejemplo: el input y el output utilizan tanto representaciones centrales como procesos diferentes, según se concluye de la exploración de pacientes que pueden leer un tipo de material que no pueden escribir y sí pueden escribir aquel que no pueden leer (RG paciente de Beauvois y Derouesné, 1981). Estas dos conclusiones van totalmente en contra de intuiciones inteligentes, puesto que intuitivamente se asumiría en el primer ejemplo la existencia de un solo procesamiento de análisis de palabras $y$, en el segundo ejemplo, una sola representación central del input y del output. Trabajando únicamente en base a intuiciones inteligentes, estaríamos en el mismo punto que Déjerine en 1892, cuando concluyó que el proceso ortográfico implicado en la lectura y la escritura era uno y el mismo.

¿Podemos entonces afirmar que la NPC, tal como está planteada en la actualidad, logrará su objetivo de conocer el funcionamiento normal? No creo que nadie pueda ni deba responder tajantemente, ni afirmando, ni negando. En realidad, la NPC puede ser considerada como un camino válido, pero en absoluto exento de problemas. A ellos vamos.

El problema más serio a primera vista es la imposibilidad de replicación. La aproximación de Morton y Patterson (1980), por ejemplo, como se ha visto, no da lugar a considerar ninguna posibilidad de replicación, puesto que si cada paciente es tratado como una potencial teoría y como un modelo de síntomas únicos, se hace imposible replicar cualesquiera de las observaciones documentadas en otro paciente. No sorprenderá al lector que Caramazza (1986) apoye totalmente este punto de vista arguyendo que la imposibilidad de replicar carece de importancia en la investigación neuropsicológica, frente al hecho de poder dar cuenta de un amplio conjunto 
de datos, ya que el peso individual que se otorgue a los resultados aislados depende del conjunto total de evidencia que tengamos a nuestra disposición.

A pesar de este y otros parecidos argumentos; tendientes a minimizar la importancia de la replicabilidad en NPC, es evidente que los fenómenos científicos replicados o replicables darían mayor peso a las conclusiones que de ellos se obtuviese. La postura epistemológica de autores como Morton y Patterson (al menos en su trabajo de 1980), o bien de Caramazza y McCloskey (1988), encierra el riesgo de llevar la investigación hacia el abandono de todo intento de generalización empírica, que no dependa totalmente de la teoría que supuestamente debe confirmar.

No obstante, vale la pena aclarar que en el punto de la replicabilidad - ya no en términos de juicio valorativo en relación con la neuropsicología, sino en términos de juicio valorativo en relación con la neuropsicología, sino en términos de simple posibilidad o imposibilidad de ser realizada en NPC - existe una clara disparidad de opiniones debido, me parece, a la ambigüedad terminológica. Los autores que consideran que la replicabilidad es imposible en neuropsicología (Shallice, 1988, por ejemplo) toman en consideración la totalidad de un caso clínico, es decir, la organización cerebral premórbida, la lesión anatómica, la alteración comportamental y todos los datos inherentes al paciente, como son el sexo, la edad, el nivel de escolaridad, etc. Frente a ello, los autores que afirman que es posible replicar en neuropsicología (por ejemplo, Allport en comunicación personal, Oxford, 1989), delimitan la replicación a los síntomas alterados presentes en más de un paciente.

Quizá una de las críticas más redicales que se han dirigido a la neurosicología cognitiva sea aquella que se refiere al tipo de notación básica, esto es, a los modelos, y muy especialmente a la falta de solidez que conllevan las "cajas y flechas" que forman los imprescindibles diagramas de flujo. El porqué de este tipo de críticas radica en el hecho de que cualquier nuevo patrón de dísociación que se encuentre, podrá ser incorporado en los modelos mediante una proliferación trivial de componentes.

Una tendencia peligrosa para la coherencia y seriedad de la NPC ha sido puesta de relieve por Ellis (1987), consistente en hacer de cada caso un síndrome. En este sentido, se encontraría el caso de Warrington y Shallice (1980) descrito de la siguiente manera: el paciente podía leer solo si, previamente a la lectura de cada palabra, pronunciaba una a una todas las letras que la componían. El paciente había perdido la capacidad de identificar globalmente las palabras, es decir, tenía dañado un subcomponente que los autores llamaron «sistema de la forma de las palabras» y, por consiguiente, concluyeron que el paciente en cuestión presentaba una «dislexia de la forma de la palabra». Esta manera de proceder parece poco deseable porque los descubrimientos que se realizan con abrumadora rapidez y difusión están constantemente modificando los hallazgos previos, por tanto, lo que hoy constituye un «síndrome» mañana ya no lo es. Este estado de cosas puede llevar a la NPC a una pérdida de credibilidad y, consiguientemente, a una pérdida de apoyo de las otras disciplinas con las que interactúa y sin las cuales difícilmente podría seguir avanzando.

Afortunadamente, no necesitamos delimitar síndromes para trabajar en NPC. En efecto, «lo único que importa es la compatibilidad o no entre patrones de sintomas y teorías cognitivas: si la teoría A explica satisfactoriamente 
los sintomas del paciente $X Y$ o debe ser el resultado de esos sintomas» (Ellis, 1987, pp. 403-404)

En resumen, entonces, tenemos que la NPC, a fin de lograr sus objetivos tendrá que adoptar el método del caso único. Dicho método, según los diferentes autores puede llevarse a cabo desde distintos planteamientos tanto epistemológicos como prácticos. Todos ellos parecen tener, al menos desde un punto de vista de clínica diaria, aciertos y desventajas. Todos ellos, por otra parte, comparten un punto crítico que en palabras muy simples quedaría expresado así: ¿qué aportan las conclusiones de estudios de caso único? Me parece que esas conclusiones pueden ayudar a comprender el funcionamiento normal siempre y cuando los estudios sean rigurosos, propongan explicaciones coherentes con el funcionamiento normal y no se limiten a ser simples descripciones de lo que un paciente hace o deja de hacer $y$, por supuesto, siempre y cuando cada caso estudiado tenga una participación en los modelos existentes, es decir, se incorpore enriqueciéndolos, ya sea falsando una teoría, descubriendo algún aspecto o poniéndolo en tela de juicio partes del modelo.

\section{Referencias}

AtKinson R. y SHIFFrin, R. (1968) Human memory: A proposed system and its control process. En K. Spence y J. Spence (Eds.), The Psychology of Learning and motivation: Advences in research and theory (Vol. 2). Nueva York: Academic Press.

Beauvols, M.F (1982) Optic aphasia: A process of interaction between vision and language. Pbilos. Trans of the Royal Soc Londres: B298, 35-47.

Beauvois, M. F. y Derouesne, J. (1981) Lexical or orthograpic agraphia. Brain, 104, 21-49.

Beauvois, M. F. , Derouesne, J. y Saillant, B. (1980) Syndromes neuropsychol. et psychologie cognitive. Trois exemples: aphasie tactile, alexie phonologique et agraphie lexicale. Cabiers de Psychologie, 23, 211-245.

Beauvois, M. F. , y Saillant, B. (1985). Optic aphasia for colour and colour agnosia: A distinction between visual and visuo-verbal impirments in the processing of colours. Cognitive Neuropsychology, 2, 1-48.

BuB, J. BuB, D. (1988) On the methodology of single case suty in neuropstchology. Cognitive Neuropsychology, 5, 565-582.

Caramazza, A. (1984) The logic of neuropsychologycal research and the problem of patient classification in aphasia. Brain \& Languaje, 19, 153-178.

Caramazza, A. (1986) On drawing inferences about the structure of normal cognitive systems from the analysis of patterns of impired performance: The case for single-patient studies. Brain and Cognition, 5, 41-66.

Caramazza, A. y Mccloskeym. (1988) The case for single-patient studies. Cognitive Neurosychology, 5, 517-528.

Сномsку, N. (1980) Rules and representations Behavioural and Brain Science, 3, 1-61.

Coltheart, M (1980) Deep dislexia: A review of the syndrom. En M. Coltheart, K. Patterson y J. Marshall (Eds.) Deep Dislexia, London: Routledge.

Coltheart, M (1986) Cognitive psychology. En M. Posner y O. Marin (Eds.). Attention and performance Hillsdale, N. J.: Lawrence Erlbaum Associates Inc.

Colgheart, M., Patterson, K., y Marshall, J. (Eds.) (1980) Deep Dislexia, London: Routledge.

COWEY, A. (1982) Sensory and non-sensory visual disorders in man and monkey. Philos Transac. of Royal Society London, B298, 3-13.

DEJERINE, J. (1892) Contribution anatomoclinique et clinique des differentes variétés de cécité verbale. Memoires de la Société de Biologie, 4, 61-90.

ElLis, A. (1987) Intimations of modularity, or, The modularity of mind. En M. Coltheart, G. Sartori y M. Job (Eds.), The cognitive neuropsychology of language. London: Lwarence Erlbaum Associates, Publishers. 
Eluis, A., y Young, A. (1988) Human Cognitive Neuropsychology, Gove and London: Lawrence Erlbaum Associates, Publishers.

Howard, D. (1987) Reading without letters? En M. Coltheart, G. Sartori y M Job (Eds.), The cognitive neuropsychology of language, London: Lawrence Erlbaum Associates Publishers.

Kinsbourne, M. (1971) Cognitive deficit: Experimental analysis. En J. L. McGough (Ed.), Psychobiology. Nueva York: Academic Press

Lakatos, I. (1964) Proofs and refutations. Bristishjournal for the Pbilosophy of Science, 14, 1-25, 120-139.

LASHLEY, K. (1929) Brain mechanisms and intelligence. Chicago: Univ. of Chicago Press

MCClOSKEY, M, y CARAMAZZA, A. (1988) Theory and methodology in cognitive neuropsychology: A response to our critics. Cognitive Neuropsychology, 5, 583-623

Mcleod, P., Mclaughling, C., y Nimmo-Smithm, I. (1985) Information encapsulation and automaticy: Evidence from the visual control of finel y turned actions. En M. Posner y O. Marin (Eds.) Attention and performance (Vol. 11) Hillsdale N. J.: Lawrence Erlbaum Associates Publishers.

MANNING, L. (1988) Neurolingüistica. Cuadernos de la UNED.

Maning, L. (en prensa) Neuropsicología cognitiva del lenguaje. En L. Manning Neuropsicología clásica y cognitiva del lenguaje: Teoría, exploración y rehabilitación de la afasia. Ed. MédicoPanamericana.

Marin, O., SAFFran, E., y Schawtz, D. (1976) Dissociations of language in aphasia: Implications for normal functions. Annals of the New York Academy of Sciences, 280, 868-884.

MARR, D. (1976) Early processing of visual information. Philosopbical Transactions of the Royal Society of London, B275, 483-524.

Marshall, J. (1986) The description and interpretation of aphasic language disorder. Neuropsychologia, 1, 5-24.

Marshall, J., y NewCOMBE, F. (1973) Patterns of paralexia: A psycholinguistic approach. Journal of Psycholinguistic Research, 2, 175-199.

Marshall, J., y Newcombe, F. (1980) The conceptual status of deep dislexia: An historical perspective. En M. Coltheart, K. Patterson, y J. Marshall (Eds.), Deep Dislexia. London: Routledge.

Morton, J. M. y Patterson, K. (1980) A new attempt at an interpretation, or, an attempt at a new interpetation. En M. Coltheart, K. Patterson y J. Marshall (Eds.). Deep Dislexia. London: Routledge.

NeISSER, U. (1981 (Memory abserved: Remembering in natural contexts. San Francisco: Freeman.

OSLON, A., y CARAMAZZA, A. (en prensa) The role of cognitive theory in neuropsychological research (se publicará). En F. Boller y J. Grafman (Eds.), Handbook of Neuropsychology. The Nederlands: Elsevier Science Publishers.

Paivio, A. (1983) The empirical case for dual coding. En J. Yuille(Ed.), Imagery, cognition and memory. Hillsdale N. J.: Lawrence Erlbaum Associates Inc.

Paivio, A. y TE LiNDE, J. (1980) Symbolic comparisosn of objects on color attributes Journal of Experimental Psychology. Human Perception and Performance, 6, 652-661.

SEINDENBERG, M. (1988) Cognitive neuropsuchology and language Cognitive neuropsychology, 6, 403-426.

SHALLICE, T. (1988) From neuropsychology to mental structure. Cambridge University Press.

SHALLICE, T. Y WARRINGTON, E. (1970) Indpendent functioning of the verbal memory stores: A neuropsychological study. Quarterly Journal of Experimental Psychology, 22, 261-273.

Teuber, H. (1955) Psychological psychology. Annual Review of Psychology, 9, 267-296.

Warrington, E. y Shallice, T. (1980) Word-form dislexia. Brain, 103, 99-112.

Weiskrantz, L. (1968) Some traps and pontifications. En L. Weiskrantz (Ed.). Analysis of behavioural change. Nueva York: Hatper $\&$ Row.

WHITACKER, H. y SLOTNICK, H. (1988) Commentes on "The case of single patient studies». Is (neuro)psychology possible? Cognitive Neuropsychology, 5, 529-534.

ZEKI, S. (1980) The representation of colouors in cerebral cortex. Nature, 18, 71-77. 